\title{
Interface roughness correlation due to changing layer period in $\mathrm{Pt} / \mathrm{C}$ multilayers
}

\author{
Amitesh Paul ${ }^{1}$ and G. S. Lodha ${ }^{2}$ \\ ${ }^{1}$ Institut für Festkörperforschung, Forschungszentrum Jülich GmbH, D-52425 Jülich, Germany \\ ${ }^{2}$ Centre for Advanced Technology, Indore 452 013, India
}

(Received 11 December 2001; revised manuscript received 11 February 2002; published 11 June 2002)

\begin{abstract}
Since the morphology in the interface plane and along the growth direction is important for imaging applications, the dependence of this on the layer period has been investigated in $\mathrm{Pt} / \mathrm{C}$ multilayers with varying period lengths $d$ ranging from 4.60 to $3.53 \mathrm{~nm}$ prepared by dc magnetron sputtering under identical deposition conditions. The Pt layer thickness in all the cases was maintained at a nominal value of $\sim 0.37 d$, and a total of 20 layer periods were deposited in each case. The grazing incidence $\mathrm{x}$-ray scattering technique has been used to study both the specular and diffuse scattering behavior of these multilayer structures. The interface roughness was found to vary from 0.35 to $0.43 \mathrm{~nm}$, and the lateral and longitudinal correlation lengths remain unchanged with a decrease in layer period as seen from the diffuse component of the scattering. However, the atomic ordering in the individual layers studied using high angle x-ray diffraction shows clearly the presence of crystallinity in the Pt layers, independent of the layer period.
\end{abstract}

DOI: 10.1103/PhysRevB.65.245416

PACS number(s): $61.10 . \mathrm{Kw}, 68.35 . \mathrm{Ct}$

\section{INTRODUCTION}

Multilayer (ML) structures of alternating high and low electron density materials with periods in the nanometer range find extensive application as optical elements for synchrotron $\mathrm{x}$ rays ranging in energy from extreme ultraviolet to hard $\mathrm{x}$ rays. They have been successfully applied to $\mathrm{x}$-ray microlithography, polarimetry, $x$-ray lasers, etc. The quality and features of the internal interfaces of MLs play a crucial role in the performances of the optical elements. Non-ideal interfaces reduce the reflectivity and produce a background halo that reduces the image contrast. The interface quality is usually determined by the magnitude and lateral character of the interfacial roughness (standard deviation of the interface height), the vertical correlation of the roughness for different interfaces, the thickness of individual layers, and the extent of intermixed layer thickness and its composition. An understanding of these interface structures are important both from technological and basic science perspectives. The study here is initiated to understand the influence of the interfacial features or the imperfections which may arise during the growth process on the overall ML quality, which in turn affects their performances.

The effect of the deposition parameters on the interface structure in $\mathrm{W} / \mathrm{Si} \mathrm{Nb} / \mathrm{Si}$, and $\mathrm{Mo} / \mathrm{Si}$ MLs has already been extensively studied and reported by various investigators. ${ }^{1-3}$ It has been found that the interface structure development is, in general, a function of the method of deposition and the deposition conditions. But apart from the effect of the deposition parameters, another important parameter that may influence the interface structure is the layer thicknesses, the effect of which has been investigated in the present work. The mechanism of multilayer growth is mostly dependent upon the evolution of the roughness at the interface and its nature along the whole stack of the ML. The roughness is widely dependent upon (i) the number of bilayers $(N)$, (ii) the value of the thickness of the period $(d)$, and (iii) the $\Gamma$ ratio defined by $d A /(d A+d B)$, where $d A$ and $d B$ are the respective thicknesses of the individual layers $A$ and $B$ and $(d A+d B)=d$, the bilayer period length. It may be noted that the propagation of roughness is quite different in case of a single-layer thin film and that of a multilayer of the same thickness. This is mainly due to the influence of the interfaces in a multilayer. Thus the effect of increasing $N$ is essentially an increase in the total thickness of the multilayer stack, keeping the individual layer thickness the same, and thus the roughness propagation due to an increasing layer thickness is not observed. Similarly by changing the $\Gamma$ ratio for fixed $d$, one increases the thickness of one layer at the expense of the other, thus it is again different from the situation of an increase in the layer thicknesses. In an earlier study by Fullerton et $a l^{2}$ it has been seen that for multilayers of $\mathrm{Nb} / \mathrm{Si}$ grown by magnetron sputtering at higher pressure there is a dramatic change in roughness as the number of layers increases. But this work does not necessarily bring out the mechanism of multilayer growth, since the effect was as a result of an extrinsic change of pressure in the deposition chamber. Very recently Freitag and Clemens ${ }^{4}$ have reported an increase of lateral correlation lengths with increasing number of layer periodicity or increasing $\Gamma$ ratio in the Si/Mo multilayer, which is again eventually an effect of the increase in the thickness of the multilayer stack as a whole. Thus the real contribution due to the increasing thickness of individual layers was not explored. Moreover the change in correlation lengths found in this study were rather small considering the large extent of errors involved in extracting the quantitative information from the experimental data. Even if the observations are considered real, this kind of behavior is to be looked for in other multilayer systems, e.g., $\mathrm{Pt} / \mathrm{C}$ where the chemical interaction of the elements used is small while the electron-density contrast is large. Thus it was necessary to study the effect of changing layer thickness or rather changing the thickness of the layer period (and not the number of periods) on the interfacial features and its correlative behavior on the overall multilayer structure in detail.

The platinum-carbon $(\mathrm{Pt} / \mathrm{C}) \mathrm{ML}$ has been widely known for $\mathrm{x}$-ray applications. ${ }^{5,6} \mathrm{Pt} / \mathrm{C}$ multilayers with high reflectivity $(\sim 5 \mathrm{~nm}$ at normal incidence) are suitable for soft $\mathrm{x}$-ray 

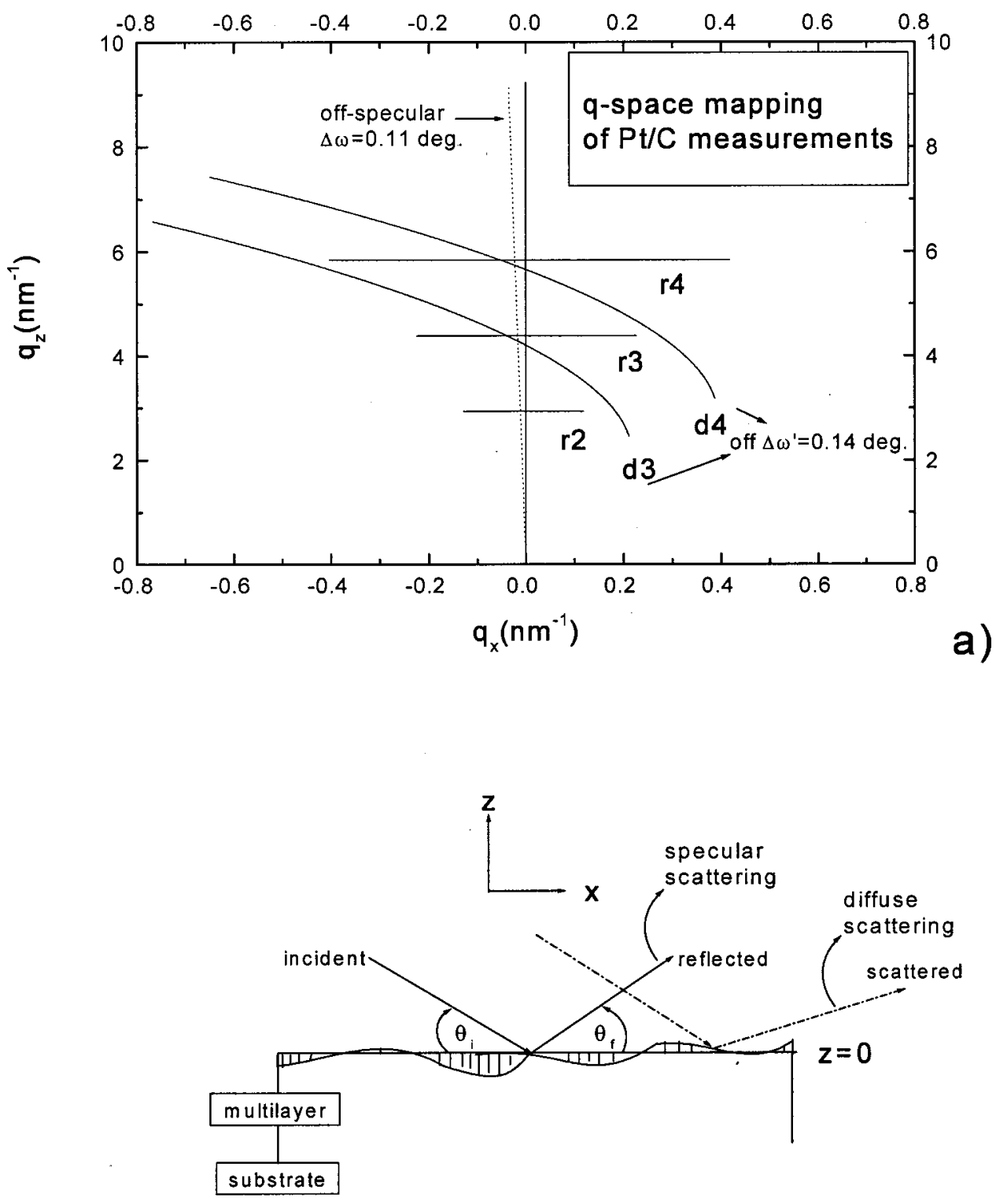

b)

FIG. 1. (a) Scans in reciprocal space $\left(q_{x}, q_{z}\right)$. The transverse scan $(r$ line), the specular (solid line), off-specular (dotted line) with an offset of $0.11^{\circ}$, and the detector scan ( $d$ line) with an offset of $0.14^{\circ}$ are all shown in the map. (b) Shows the scattering geometry at grazing incidence and exit angles in real space.

mirrors due to the smaller absorption coefficient of $\mathrm{C}$ around the wavelength regime; therefore in the present work $\mathrm{Pt} / \mathrm{C}$ MLs were chosen for investigation.

In recent years the usage of $\mathrm{x}$-ray scattering measurements in specular and nonspecular geometries have been effectively explored for characterization of interfacial structures in MLs. ${ }^{7}$ One may note the different regimes of scans in $q$ space for measurements done in the present case in Fig. 1(a). Following the diagram one can see the dependence of $q_{x}$ and $q_{z}$ components in different scans. We define the momentum-transfer vectors $q_{x}$ and $q_{z}$ in the direction parallel and perpendicular to the film plane, respectively, the incident angle and the exit angle with respect to the film surface being $\theta_{i}$ and $\theta_{f}$. The dependence of $q$ in-plane and perpendicular to the plane directions can be expressed as $q_{x}=K\left(\cos \theta_{f}-\cos \theta_{i}\right)$ and $q_{z}=K\left(\sin \theta_{i}+\sin \theta_{f}\right)$, where $K$ $=2 \pi / \lambda$ with a wavelength $\lambda$. Figure $1(\mathrm{~b})$ shows the scattering geometry of a monochoromatic beam which is incident on the surface at an angle $\theta_{i}$ and scattered at $\theta_{f}$. In specular geometry, $q_{i}=q_{f}$ and is a scan along the $q_{z}$ axis. For offspecular scans the offset is $\left(\theta_{i}-\theta_{f}\right)=\Delta \omega=0.11^{\circ}$. The rocking scans (transverse scans) are nearly $q_{x}$ scans with $q_{z} \approx$ const, as the scattering angle is fixed. Finally the detector scans are done keeping $\theta_{i}$ fixed with an offset $\left(\Delta^{\prime} \omega=0.14^{\circ}\right.$ ) angle corresponding to the minimum of the Bragg peaks and varying the detector angle $\theta_{f}$.

In the specular reflectivity measurements, wave-vector transfer is changing perpendicular to the sample surface di- 
rection. This provides the information about the individual layer thickness and an estimate of the rms interfacial roughness. But scattering of electromagnetic radiation from nonideal surfaces and interfaces results in a loss of specular reflectivity, which gives rise to nonspecular reflectivity. The incoherent (diffuse) component of the scattered intensity depends on an in-plane component of the scattering vector and reproduces the details of the lateral ordering of the interface roughness as well as the character of vertical replications of roughness from one interface to another. It may be noted that vertically correlated roughness may influence device properties differently from that with noncorrelated or uncorrelated roughness. ${ }^{8}$ In case of optical MLs the horizontal correlations strengthen the diffusely scattered intensity in the vicinity of the specular beam, and the correlation length affects the spatial distribution of the incoherent halo around the specular direction. The imaging and the reflective features of multilayers with correlated interfacial roughness change nonlinearly with regard to the operating wavelength of a mirror and the variation is parametrized by the magnitude of the roughness correlation. The strong interference effects occurring during the scattering of $\mathrm{x}$ rays from MLs due to cumulative roughness replications and the dynamical nature of the multiple $\mathrm{x}$-ray scattering within high quality ML give rise to specific patterns of diffuse intensity that contain unique information on the nature of the interfacial structures. The recorded diffuse intensity can be used to determine mesoscopic in-plane structure of surfaces and interfaces, lateral correlation of roughness, and the fractal dimension of jagged surfaces. For a multilayer structure, intensity will be weaker in the absence of vertical correlation as there will be a random phase relation between intensities scattered from each layer. But there may be lateral correlation of roughness for each layer to produce diffuse scattering with a well-defined intensity distribution in the $\left(q_{x}, q_{y}\right)$ plane, i.e., a weak dependence of the lateral interfacial parameters within the secondorder DWBA (distorted-wave Born approximation) may be present in the specular patterns. ${ }^{9}$ Thus an off-specular scan or a detector scan ( $2 \theta$ scan) can give information regarding the correlation along the film normal, coupled with that of the film plane. One may note that the longitudinal scans provide information about the correlation of interface roughness of different interfaces, while the transverse scans are sensitive to the height-height correlation function and lateral coherence length of the interface roughness. ${ }^{2}$

The x-ray reflectivity from a multilayered system is evaluated using the coherent scattering approximation developed by Parratt in $1954 .{ }^{10}$ The fittings of the x-ray reflectivity patterns were done after the subtraction of the off-specular reflectivity from the specular one to obtain the true-specular patterns. The reflectivity of the ML has been fitted by the least-squares method using the standard optical formalism taking the interface roughness into account according to Nevot and Croce. ${ }^{11}$ The incoherent component of scattered $\mathrm{x}$ rays caused by roughness is calculated by the perturbation theory using the DWBA, taking into consideration only the primary scattering process. Sinha et al. ${ }^{7}$ calculated the cross section for diffuse scattering from a single surface. They demonstrated how DWBA could be used to quantitatively understand the diffuse scattering, whereas the extension of the DWBA formalism to a multilayered system has been done by Holy and Baumbach. ${ }^{12}$ They have included the effect of correlations between the interfaces. Later on, a number of groups were involved in using the formalism with different models of roughness correlations.

In the approximation of single scattering, the scattering function can be written as

$$
S(q)=\iint \rho(r) \rho\left(r^{\prime}\right) \exp \left\{i q\left(r^{\prime}-r\right)\right\} d r d r^{\prime},
$$

$\rho$ is the electron density profile and $r=(x, y, z)$ and $r^{\prime}$ $=\left(x^{\prime}, y^{\prime}, z^{\prime}\right)$ are independent spatial coordinates.

We write $S(q)=S_{\text {specular }}(q)+S_{\text {diffuse }}(q)$, where

$$
\begin{aligned}
S_{\text {diffuse }}(q)= & \frac{(\Delta \rho)^{2}}{q_{z}^{2}} \exp \left(-q_{z}^{2} \sigma^{2}\right) \int\left[\exp \left\{q_{z}^{2} C(R)\right\}-1\right] \\
& \times \exp (i q \cdot R) d R
\end{aligned}
$$

and $\Delta \rho$ is the electron density contrast. The diffusely scattered intensity $I_{\text {diffuse }}$ from a single surface is expressed as $I_{\text {diffuse }} \sim\left|t_{i}\right|^{2} S(q)\left|t_{f}\right|^{2}$, where $t_{i}$ and $t_{f}$ are the coefficients of transmission within the layers, which take the Fresnel coefficient into account. The in-plane structure of the interfaces can be described in terms of a height-height correlation function $c(x, y)$, which in most of the cases can be written as the self-correlation function $c_{0}(x, y)=\sigma^{2} e^{-(|R| / \xi)^{2 h}}, \quad R=\left(x^{2}\right.$ $\left.+y^{2}\right)^{1 / 2}$, where the parameters are the rms interface roughness $\sigma$, the fractal dimension $h$, which takes care of the jaggedness, the longitudinal correlation length $\kappa$ (vertical distance over which correlations decay to 1/e of the rms value), and the lateral correlation length $\xi$ (horizontal distance over which correlations decay to $1 / e$ of the rms value). ${ }^{13}$

Some basic models for the roughness cross correlation within the multilayers have been reported in the literature. ${ }^{12-16}$ The models are developed based on the different possibilities of roughness correlation along the multilayer structure. In our study we have tried different mechanisms of vertical and in-plane roughness correlation with the models in Refs. 12, 14, and 15. Holy and Baumbach's model ${ }^{12}$ is a complete correlation model. It takes into account that interfaces are formed successively from the substrate to the surface. Each interface adds some statistically independent roughness, which is assumed in this model to be completely transferred to all the successive interfaces. Thus, the roughness is accumulated. The correlation between two interfaces is determined by the contributions of all the interfaces below the lower one because the roughness added between the lower and higher interfaces is independent of the roughness of the lower interface. It may be noted that the rms roughness specified for this model is the incremental roughness. The model of Spiller, Stearns, and Krumrey ${ }^{15}$ also derives the respective correlation function for the diffuse scattering simulations. This model assumes the accumulation of roughness like in Holy's model, but the roughness added at each interface is not completely inherited by successive interfaces. The inheritance is lower, the shorter the lateral size of roughness is. As a result, the lateral size of total roughness 
grows towards the surface even if all the interfaces add roughness with the same size. The total rms height may increase or decrease towards the surface depending on whether the accumulation or dissociation of roughness is dominating. The model of Ming et al. ${ }^{14}$ on the other hand describes an intermediate case between uncorrelated roughness [the roughness of different interfaces is not correlated and each interface is assumed to possess fractal (self-affine) roughness with the correlation function by Sinha et al. $\left.{ }^{7}\right]$ and completely correlated roughness (similar to uncorrelated, but the roughness of different interfaces is assumed to be completely correlated or conformal). The model assumes that vertical correlation does not depend on the lateral size of roughness. Here the replication factor is controlled by the vertical correlation length $\kappa$ and the correlation function is given by $c(x, y)=\sigma^{2} c_{0}(R) e^{-(|z| / \kappa)}$, where $c_{0}$ is the self-correlation function and $z$ the coordinates of interfaces. Thus roughness can vary from $\kappa=0$ to $\kappa$ much greater than the thickness of the ML.

However, no parameter set could be obtained in order to bring the simulated and measured profiles into agreement for the set of samples either with the model of Ref. 12 or with that of Ref. 15. This may be due to the fact that the replication of roughness across layers is neither incremental nor accumulative but of the 'restarting layer' type ${ }^{4}$ behavior due to the presence of $\mathrm{C}$ at each interface. A good agreement can only be arrived at with the model of Ming et al., where the information obtained in-plane and out-of-plane is decoupled.

\section{EXPERIMENTAL DETAILS}

$\mathrm{Pt} / \mathrm{C}$ MLs with different period lengths $d$ ranging from 4.60 to $3.53 \mathrm{~nm}$ were made on a float glass substrate, kept at room temperature, by dc magnetron sputtering specially designed for coating the inner walls of the cylindrical surfaces. Two sputter sources of Pt and C were located at the top and bottom of the cylindrical vacuum chamber. All samples were grown at a low Ar pressure of $1 \mathrm{mbar}$. The deposition rate for $\mathrm{Pt}$ and $\mathrm{C}$ was 0.1 and $0.04 \mathrm{~nm} / \mathrm{sec}$, respectively. The layer thickness during deposition was controlled by the ion current and the deposition time. Uniformity in the horizontal plane is achieved by rotating the sample, while the mask achieves vertical uniformity. The overall thickness uniformity was found to be $<2 \%$ over an area of $10 \times 10 \mathrm{~cm}^{2}$. The thickness of individual layers was controlled to within $0.1 \mathrm{~nm}$. Multilayers with the same parameters are reproduced within $1 \%$ accuracy. The Pt layer thickness in all the cases was maintained at a nominal value of $\sim 0.37 d$ (i.e., the thickness ratio $\Gamma=0.37$ ), and a total of 20 layer pairs were deposited in each case.

Specular and nonspecular diffused scattering at wavelength $\lambda=0.154 \mathrm{~nm}(\mathrm{Cu} K \alpha)$ were measured on a x-ray diffractometer with a grazing incidence attachment and a sealed tube with a $\mathrm{Cu}$ target. The scattered beam divergence is $\sim 0.4^{\circ}$ defined by soller slits. The scattered beam is monochromatized using a LiF (110) crystal placed before the NaI (Tl) scintillation detector. The $\mathrm{x}$-ray scattering behavior was studied by specular $(\theta-2 \theta)$ scans, longitudinal offset $(\theta-2 \theta$ $+\Delta \omega)$ scans, rocking $(\omega)$ scans, and detector $(2 \theta)$ scans. The

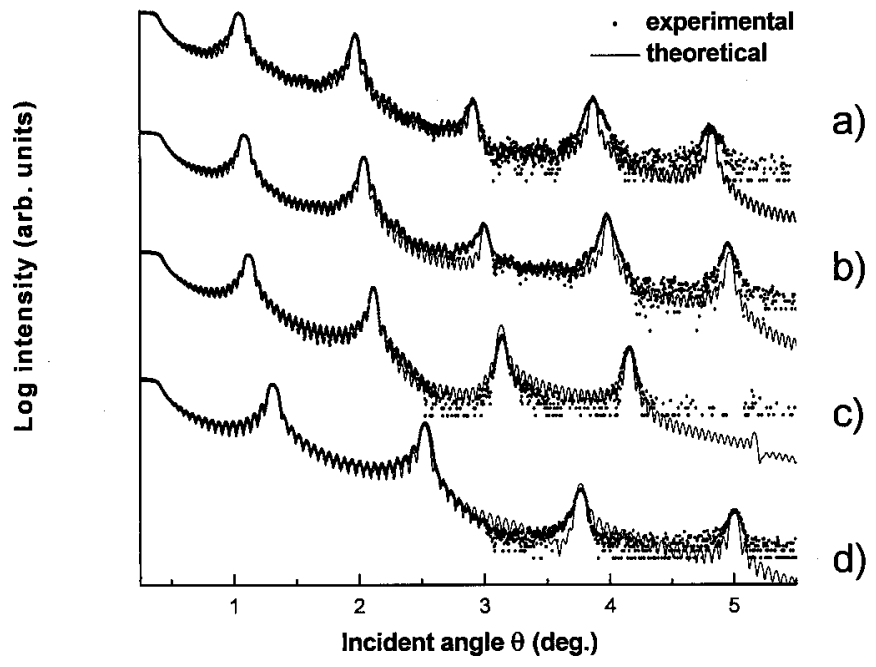

FIG. 2. The true-specular scans along with the best simulated curve corresponding to the four MLs is shown as a function of angle of incidence. The different $d$ values corresponding to $a, b, c$, and $d$ of the MLs and the parameters from the fit to the curves are reported in Table I. The curves are vertically shifted for the sake of clarity.

presence of atomic order in the individual layers was studied by $\mathrm{x}$-ray diffraction (XRD) at high angles. The XRD spectra were recorded with a fixed angle of incidence of $0.5^{\circ}$ (to limit the penetration of the beam to film thickness) and the detector is scanned from $20^{\circ}$ to $80^{\circ}$.

\section{RESULTS AND DISCUSSION}

Low angle x-ray diffraction scans were taken to measure both the diffuse and specular scattered intensities. The scattered intensity is measured in the specular geometry $(\theta-2 \theta)$ and off-specular $\left(\theta-2 \theta+0.11^{\circ}\right)$ geometry for the four different MLs. Figure 2 shows the true-specular (specular minus off-specular) reflectivity patterns along with their fit. The simulation results are shown in Table I. In addition to the well-defined Bragg peaks up to the fifth order ( $n=1$ to 5$)$, the presence of well-defined finite-thickness higherfrequency oscillations (Kiessig fringes) clearly indicates the presence of a well-ordered layered structure irrespective of

TABLE I. Pt/C ML reflector parameters obtained from XRR and XDS patterns.

\begin{tabular}{|c|c|c|c|c|c|}
\hline Sample & $\begin{array}{c}\Gamma \\
( \pm 0.01)\end{array}$ & $\begin{array}{c}d(\mathrm{~nm}) \\
( \pm 0.01 \mathrm{~nm})\end{array}$ & $\begin{array}{c}\sigma(\mathrm{nm}) \\
( \pm 0.01 \mathrm{~nm}) \\
\text { from specular } \\
\text { scan at } \\
\text { interfaces of } \\
\mathrm{Pt}-\mathrm{C} / \mathrm{C}-\mathrm{Pt}\end{array}$ & $\begin{array}{c}\kappa(\mathrm{nm}) \\
\pm 20 \mathrm{~nm} \\
\text { from off- } \\
\text { specular } \\
\text { scan }\end{array}$ & $\begin{array}{c} \\
\sigma_{c}(\mathrm{~nm}) \\
( \pm 0.1 \mathrm{~nm})\end{array}$ \\
\hline$a$ & 0.34 & 4.60 & $0.41 / 0.35$ & 150 & 0.4 \\
\hline$b$ & 0.34 & 4.48 & $0.41 / 0.35$ & 150 & 0.4 \\
\hline$c$ & 0.38 & 4.27 & $0.41 / 0.41$ & 150 & 0.4 \\
\hline$d$ & 0.37 & 3.53 & $0.41 / 0.43$ & 150 & 0.4 \\
\hline
\end{tabular}


the variation in $d$ or the ML period. One may note that the Bragg-peak widths are seen to increase with increasing order of reflection, which is basically a signature of the cumulative nature of the disorder as observed also by Fullerton. ${ }^{17}$ The ML period $d$ can be determined accurately from the position of the Bragg peaks and is found to be 4.60, 4.48, 4.27, and $3.53 \mathrm{~nm}$, respectively, for MLs A, B, C, and D. The limited divergence of $0.02^{\circ}$ is taken into consideration in the specular reflectivity simulations. The substrate bending due to deposition and the associated curvature effects are not particularly relevant in our case, as the ML were deposited on 3.0-mm-thick float glass substrates. We have not considered the thickness of the interdiffused layer, as negligible intermixing is expected from the two elements $(\mathrm{Pt}$ and $\mathrm{C})$ with a positive heat of mixing. A difference in Pt-C and C-Pt interface has been observed to give a significant improvement to the fit, while the Pt-C interface was found to remain similar for all the MLs unlike the C-Pt interface. The fitting was done keeping the individual refractive indices of the bulk $\mathrm{Pt}$ and $\mathrm{C}$ and the interface roughness of each alternative interface equal. The thickness of the individual layers, $\mathrm{Pt}$ and $\mathrm{C}$, and the average interface roughness $\sigma$ in each of the MLs has been determined from these fitted patterns. It can be seen from the specular reflectivity results that there is a little decrease in the average interface roughness $\sigma$ at the C-Pt interface as the $d$ spacing increases. This decrease in $\sigma$ with $d$ is attributed to the $\mathrm{C}$ layer, which causes a smoothening effect at each C-Pt interface.

Interface roughness is often assumed to scale with the number of layers deposited, where the two elements used are indistinguishable, i.e., similar in Z. But when there is a possibility that roughness depends upon the interdiffusion or chemical reaction, then the interfacial roughness would be relatively dependent upon the bilayer period also. Since the interfacial roughness is controlled by the evolution of individual layer morphologies, i.e., individual grain sizes, which are more prominent for shorter periods, the bilayer period should also have a strong effect on roughness. This is why short period x-ray mirrors are found to be inefficient reflectors. ${ }^{18}$ In the present case if the evolution of roughness is estimated following the growth law. ${ }^{19} \varpi$ (interface width/ rms roughness) $\propto t$ (thickness) ${ }^{\beta(\text { growth exponent })}$, then following Table I, a roughness change from 0.35 to $0.43 \mathrm{~nm}$ gives $\beta$ as $\sim 0.1$. This is indeed very small to explain the effect of the period. This smallness is due to the suppression of the interface roughness due to the presence of interfaces. This suppression would not have been observed if a single layer of similar thickness has been deposited. It may be noted that the error bars used in the fitting are significantly low compared to the values reported in the previous works. ${ }^{12}$ This accuracy is because of the sharp contrast in the electron densities of the two elements used for deposition and the interfacial sharpness.

As each layer is deposited on the outer surface of the prior layer, there is a possibility that the roughness from one layer surface to the next be replicated in some manner, which is a measure of the correlation of the stack and thus is dependent on the layer period. To observe the extent of correlation, the off-specularly scattered intensity has been observed over the

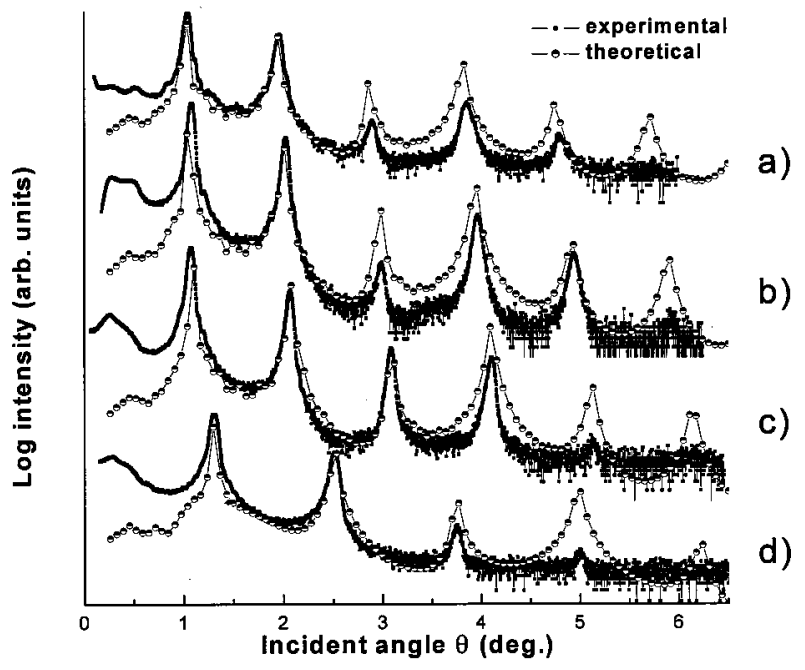

FIG. 3. The off-specular scans along with the best simulated curve corresponding to the four MLs is shown as a function of angle of incidence. The parameters from the fit to the curves for different $d$ values corresponding to $a, b, c$, and $d$ of the MLs are reported in Table I. The curves are vertically shifted for the sake of clarity.

entire range of $q$ space that was available in the experimental setup used. Figure 3 gives the plot of the off-specular scan along with their fit. The fitted parameters are tabulated in Table I. From the off-specular spectra it can be seen that the longitudinal diffuse spectra of all the different multilayer structures are peaked at the same $q_{z}$ values as the specular ones. This indicates that there exists at least a partial vertical correlation from layer to layer. However the off-specular scans in the present case give a roughness value of $0.4 \mathrm{~nm}$ and the vertical correlation $\kappa$ as $150 \mathrm{~nm}$, whereas the lateral correlation parameter $\xi$ and jaggedness $h$ values are found to be not sensitive enough to the simulations. In a multilayer structure, a part of the interface height variation is correlated between successive layers, while another part is uncorrelated or random. Following the correlation relation $\left(\sigma_{\text {random }}^{2}\right.$ $\left.=\sigma_{\text {total }}^{2}-\sigma_{\text {corr }}^{2}\right), \sigma_{\text {random }}$ for all MLs was found similar. Here interface roughness $\sigma$ has been designated as $\sigma_{\text {total }}$. This result does agree with the previous observation in W/C multilayers by Savage et al., ${ }^{13}$ where an increase in the total roughness $\left(\sigma_{\text {total }}\right)$ in the multilayer with increasing layer period was observed but $\sigma_{\text {random }}$ was found similar. But their observation of vertical correlation lengths changing as inferred from the transverse scan profiles may not be satisfactory, since the vertical correlation parameter is more effectively reflected in the longitudinal scans rather than in transverse scans where the lateral scan is the sensitive parameter.

In order to verify the present results inferred from the off-specular scans, the detector scans were also done in an offset geometry, keeping the angle of incidence at an offset from the position of the third- and fourth-order Bragg peak. Figure 4 shows the detector scan spectrum for the MLs of different periodicities corresponding to the third- and fourthorder Bragg peaks with an offset angle of $0.14^{\circ}$. As seen in 

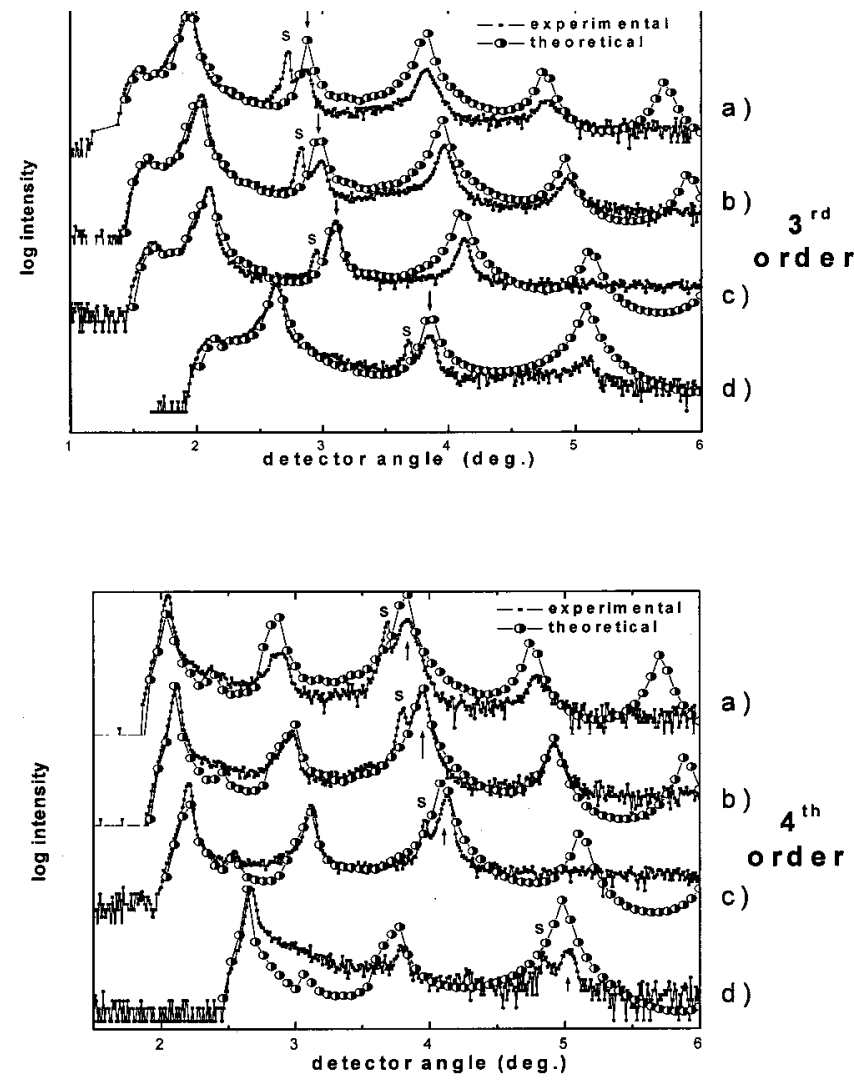

FIG. 4. The diffuse intensity streaks at the different Bragg positions are measured by the $2 \theta$ detector scans. The angle of incidence in these scans is kept at an offset of $0.14^{\circ}$ from the thirdorder and fourth-order Bragg-peak position. The parameters from the fit to the curves for different $d$ values corresponding to $a, b, c$, and $d$ of the MLs are reported in Table II. The arrows indicate the position of the diffuse Bragg streaks and $S$ denotes the position of the specular beam. The curves have been vertically shifted for clarity.

these scans the ratio of the specular to off-specular intensity peaks clearly shows the increased diffuse scattering component for the scans measured at the third- and fourth-order Bragg peaks, and the diffuse part is more pronounced in case of the fourth-order Bragg position than that of the third order. The fit to the data is reported in Table II. The results are in

TABLE II. Pt/C ML reflector parameters obtained from optical simulations for XDS for different samples measured at different $q_{z}$ values corresponding to second-, third-, and fourth-order Bragg peaks.

\begin{tabular}{ccccccc}
\hline \hline & \multicolumn{2}{c}{ Rocking scan } & & \multicolumn{2}{c}{ Detector scan } \\
\cline { 2 - 3 } \cline { 5 - 6 } Sample & $\begin{array}{c}\xi(\mathrm{nm}) \\
( \pm 3.0 \mathrm{~nm})\end{array}$ & $\begin{array}{c}h \\
( \pm 0.1)\end{array}$ & & $\begin{array}{c}\kappa(\mathrm{nm}) \\
( \pm 20 \mathrm{~nm})\end{array}$ & $\begin{array}{c}\sigma(\mathrm{nm}) \\
( \pm 0.1 \mathrm{~nm})\end{array}$ \\
\hline$a$ & 12.0 & 0.5 & & 150 & 0.4 \\
$b$ & 12.0 & 0.5 & & 150 & 0.4 \\
$c$ & 12.0 & 0.5 & & 150 & 0.4 \\
$d$ & 12.0 & 0.5 & & 150 & 0.4 \\
\hline \hline
\end{tabular}

agreement with that of the off-specular scans in Fig. 3.

The diffuse scattering from the MLs measured by the transverse rocking scans or $q_{x}$ scans for different values of $q_{z}$ are shown in Fig. 5 taking into account the asymmetry correction. ${ }^{13}$ The rocking scan was measured at the second-, third-, and fourth-order superlattice Bragg peak position, i.e., for different $q_{z}$ values in all the four different MLs. The scan contains the specular peak at $q_{x}=0.0 \mathrm{~nm}^{-1}$ and a broad diffuse component below as the background of the specular peak. The shape of the diffuse component depends on both the lateral coherence length and the details of the heightheight correlation function needed to characterize the interfacial roughness. For the larger values of $q_{x}$, the transverse scan is more sensitive to the high-frequency corrugation of the interfacial roughness. ${ }^{16}$ However from the fit to the data, the values for lateral $(12 \mathrm{~nm})$ and longitudinal correlation lengths $(150 \mathrm{~nm})$ were found independent of the layer periodicity and also of the $q_{z}$ values, i.e., for the second-, third-, and fourth-order Bragg peaks the correlation lengths as well as the jaggedness were the same. This indicates that the interfacial roughness is correlated for all the frequencies explored. The width of the diffuse component increases with $q_{z}$, which gives an estimation of the lateral length scale of the vertically correlated roughness from the rocking curve measurements. This length is typically of the order of other crystalline/amorphous multilayers. ${ }^{20}$ The values obtained are given in Table II. These results show that the roughness correlation along the interface plane does not change even as the period $d$ decreases.

One may note that in case of the top panel of Fig. 5 ( $n$ $=2$ ), though one is geometrically blocked from measuring scattered intensity due to small wavelength roughness, yet that small wavelength roughness is still seen by the x-ray beam and influences the scattered $\mathrm{x}$-rays. Whereas in case of the bottom panel $(n=4)$, one is well within the angular range where one can measure such a length scale. It may be noted that lateral correlation lengths are best estimated from the spectra of the bottom panel (higher order $n$ ). ${ }^{21}$ Therefore for lower orders the scattered intensity measured is fitted to a model that is valid for wider length (which can be upto its limiting value $\xi_{\text {limit }}=2 \pi / q_{x(\max )}$ ) than one can measure (so in a sense, one is extrapolating). As long as most of the scattered intensity is in the range one is measuring, it is fine. Moreover it may also be noted that the measurements at higher order are less surface sensitive because of the changing path length in the sample, ${ }^{21}$ and since the interfacial roughness in this case is assumed not to change through the film, and one does not even see any oxide formation near the surface (as inferred from the x-ray reflectivity patterns), therefore it may be extrapolated to lower orders. The apparent flatness in the top panel of Fig. 5 may be seen as due to the restricted geometry involved and not due to any change in the 12-nm correlation. The sharper peak or enhanced scattering around the specular reflection is most prominent in the top panel and specially in case of sample $a$, where the angular range is most restricted and the specular intensity is most intense and the scattered intensity is not distributed over the wider range as in the bottom panel.

Very recently Freitag and Clemens have reported ${ }^{4}$ an in- 

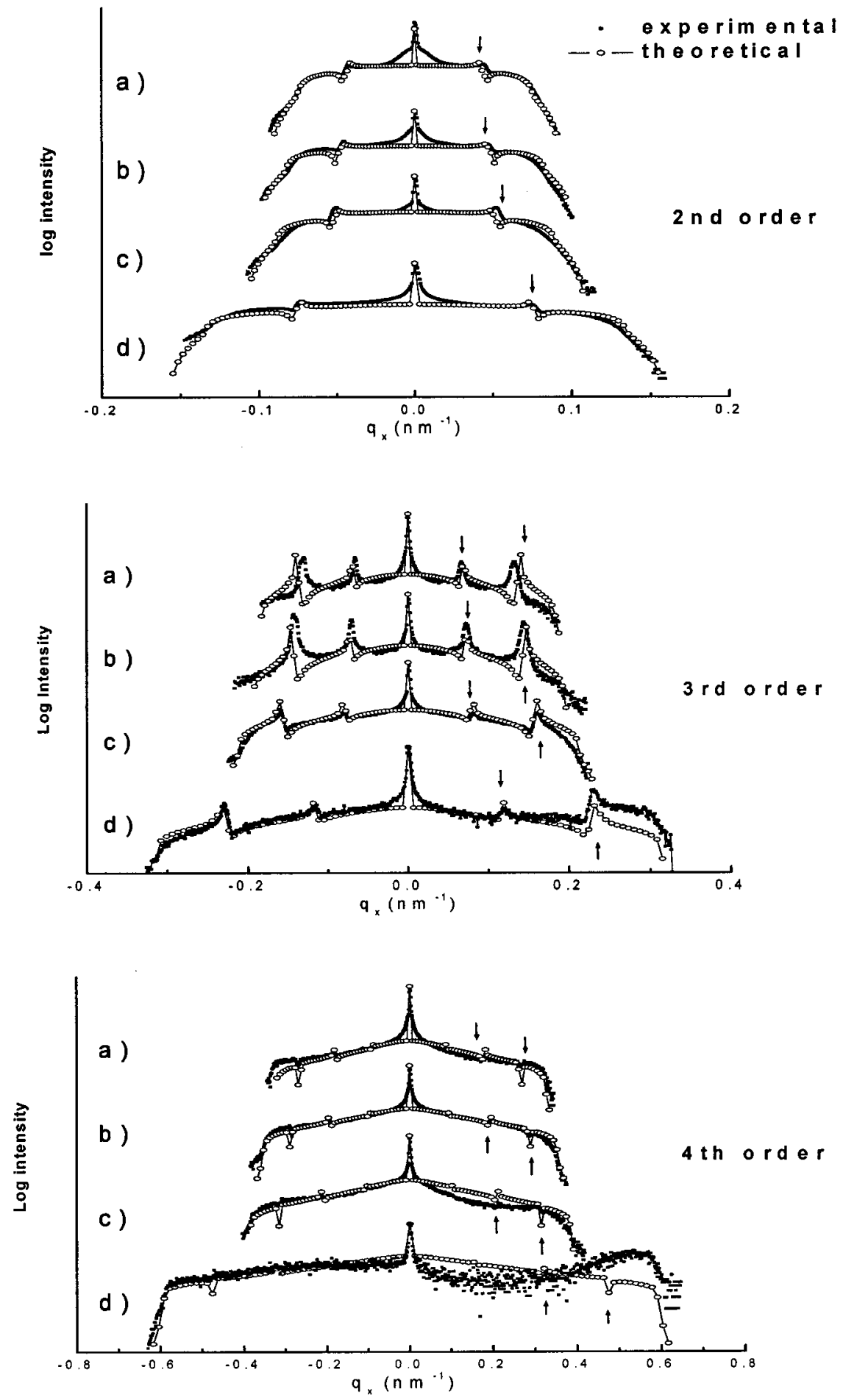

FIG. 5. The transverse rocking scans at the second-, third-, and fourth-order Bragg peak indicate the nature of interface morphology present in the different MLs. The parameters from the fit to the curves for different $d$ values corresponding to $a, b, c$, and $d$ of the MLs are reported in Table II. The arrows indicate the position of peaks at $\theta_{i} / \theta_{e}=\theta_{n B}$. The curves have been vertically shifted for clarity. Open circles show the fitted curves. crease in the lateral correlation lengths $(\xi)$ with increasing number of bilayers in $\mathrm{Si} / \mathrm{Mo}$ multilayers systems. This is very much in contradiction to our results in $\mathrm{Pt} / \mathrm{C}$ multilayers. According to their results one may figure out two observations: (1) With a fixed ratio of layer thickness (0.4), a change in the number of layer periods $(N)$ from 5 to 40 causes the correlation length to change from 1.9 to $5.2 \mathrm{~nm}$, which is a change of only $3.0 \mathrm{~nm}(2)$ For a fixed $d(d=20)$ the change in $\Gamma$ ratio from 0.2 to 0.8 has changed the correlation length by $8.0 \mathrm{~nm}$. Therefore the changing $\Gamma$ ratio with a fixed period has more effect on correlation parameters than the effect of the increase in individual layer thicknesses. In comparison, the present results in $\mathrm{Pt} / \mathrm{C}$ multilayers show a change of $\sim 1.1$ $\mathrm{nm}$ in the thicknesses of $d$ which is effectively a change of $(1.1 \times 20) \sim 20 \mathrm{~nm}$ in total thickness but has caused no variation in $\xi$. For a similar change in total thickness for $\mathrm{Si} / \mathrm{Mo}$ MLs the change in $\xi$ was only $\sim 0.5 \mathrm{~nm}$. This change in $\mathrm{Si} / \mathrm{Mo} \mathrm{MLs} \mathrm{is} \mathrm{quite} \mathrm{insignificant} \mathrm{and} \mathrm{is} \mathrm{expected} \mathrm{due} \mathrm{to} \mathrm{the}$ high degree of errors involved in the measurements. ${ }^{12}$ Thus it can be said that, keeping the $\Gamma$ ratio unchanged, the indi- 
vidual thicknesses of the multilayered elements does not change the correlation parameters within the multilayer. These results cannot be explained in the light of the arguments by Savage et al., ${ }^{13}$ since the interfaces has played no part in preferential smoothening of only the high frequency roughness. This can be argued because there was no change in vertical as well as the lateral correlation lengths with the period of the MLs, thus both the short and long wavelength roughnesses are replicated through the multilayer stack. The smoothening effect though explains the increase in interface roughness with decreasing layer thickness. It may be noted that the quality of the data presented in this letter is comparable to that of the synchrotron data by Freitag and Clemens. ${ }^{4}$ The data are measured and analyzed for all ranges of frequencies and for correction factors absorption at low incident angles as well as the larger footprint at low incident angle have been taken into account while simulating the profiles.

The diffuse scattering spectra measured by the rocking scans also show symmetrical sharp intensity modulations on either side of the specular peak as seen for the second-, third-, and fourth-order Bragg peaks in Fig. 5. The positive Bragg streaks and the negative ones are due to the dependence of the different phase relationships (constructive and destructive) between the incident and scattered intensities existing at the lower and higher $q_{z}$ values, respectively. ${ }^{22-24}$ When the angle of exit $\theta_{e}$ approaches $\theta_{n B}$ (Bragg angle) the coherent scattering from the randomly arranged atoms acts as a source of $\mathrm{x}$ rays inside the ML and on interference with the one-dimensional ML gives rise to sharp intensity modulations (Kossel lines). ${ }^{25}$ In either case the coherent scattering from the amorphous state of individual layers is predicted to interfere with the one-dimensional ML crystal. The presence of amorphous order in the individual $\mathrm{W}$ and Si layers has been confirmed by electron microscopy. In the present work the presence/absence of amorphous order in the layers was studied by high angle $\mathrm{x}$-ray diffraction (XRD) at grazing angles of incidence. Figure 6 clearly shows the presence of crystalline order in the Pt layers. The well-defined Pt(111) and $\operatorname{Pt}(220)$ peaks can be seen in the diffraction spectrum for multilayers where the period $d=4.60$ and $4.27 \mathrm{~nm}$. The corresponding grain size as obtained from the XRD spectra evaluated from the Scherrer formula along the $q_{z}$ direction is around $1.5 \mathrm{~nm}$ for both the $d$ values. These results clearly show that sharp intensity modulations observed in the transverse rocking scans in Fig. 5 are not due to coherent scattering from the amorphous state of individual layers. The coherent scattering from an ordered arrangement of atoms in the Pt layer acts as a source to excite other scattering processes. Such a scattering behavior is also observed in long period AlAs/GaAs multilayers. ${ }^{12}$

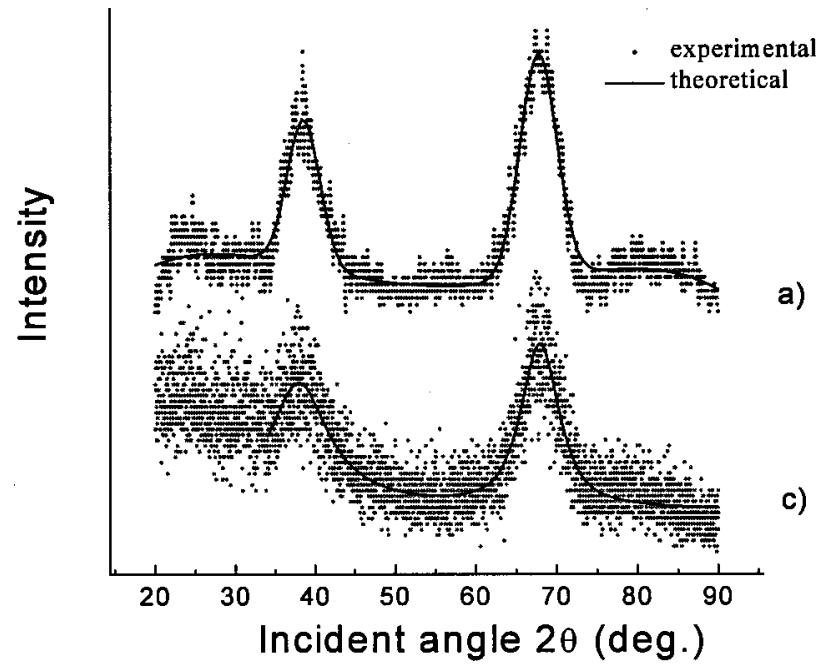

FIG. 6. The high angle XRD spectrum performed with $\lambda$ $=0.154 \mathrm{~nm}$. X-rays show the presence of crystalline Pt in the MLs for sample (a) $d=4.60 \mathrm{~nm}$ and (c) $d=4.27 \mathrm{~nm}$. The angle of incidence was kept at $0.5^{\circ}$ in order to limit the depth of penetration of the incident beam to the film thickness. The solid line is a fit to the data using a psuedo-Voigt profile.

\section{CONCLUSIONS}

The morphological development of interfaces in $\mathrm{Pt} / \mathrm{C}$ multilayers has been studied as a function of the thickness of the multilayer period $d$. The interface roughness has increased by a small amount with increasing $d$ but is found to be correlated both in the plane of the multilayer and along the growth direction even at large $d$. The roughness correlations were found to remain unchanged even as the $d$ values decreases from 4.65 to $3.50 \mathrm{~nm}$ and also were constant for different ranges of $q_{x}$. The diffuse scattering from all the MLs exhibit intensity modulations or streaks in the radial scan direction, which are positive and negative as well. These intensity modulations were found to be due to the coherent scattering from the periodic arrangement of atoms in the Pt layer and not due to the scattering from randomly distributed atoms as predicted earlier.

\section{ACKNOWLEDGMENTS}

The authors are thankful to Professor Ajay Gupta of IUC-DAEF (Indore), India for his cooperation in using the $\mathrm{X}$-ray diffractometer and useful discussions during the work. Thanks are also due to the Department of Physics, Nagoya University, Japan for helping in sample preparation.
${ }^{1}$ T. Salditt, D. Lott, T. H. Metzger, J. Peisl, G. Vignaud, P. Hoghoj, O. Scharpf, P. Hinze, and R. Lauer, Phys. Rev. B 54, 5860 (1996).

${ }^{2}$ E. E. Fullerton, J. Pearson, C. H. Sawers, S. D. Bader, X. Z. Wu, and S. K. Sinha, Phys. Rev. B 48, 17432 (1993).

${ }^{3}$ S. P. Vernon, D. G. Stearns, and R. S. Rosen, Appl. Opt. 32, 6969 (1993).

${ }^{4}$ J. M. Freitag and B. M. Clemens, J. Appl. Phys. 89, 1101 (2001). 
${ }^{5}$ G. S. Lodha, K. Yamashita, T. Suzuki, I. Hatukadi, K. Tamura, T. Ishigana, S. Takahama, and Y. Namba, Appl. Opt. 33, 5869 (1994).

${ }^{6}$ G. S. Lodha, K. Yamashita, H. Kunieda, Y. Tawara, J. Yu, Y. Namba, and J. M. Bennett, Appl. Opt. 37, 5239 (1998).

${ }^{7}$ S. K. Sinha, E. B. Sirota, S. Garoff, and H. B. Stanley, Phys. Rev. B 38, 2297 (1988); T. Salditt, T. H. Metzger, and J. Peisl, Phys. Rev. Lett. 73, 2228 (1994).

${ }^{8}$ Amitesh Paul, J. Magn. Magn. Mater. 240, 497 (2002).

${ }^{9}$ D. K. G. de Boer, Phys. Rev. B 49, 5817 (1994).

${ }^{10}$ L. G. Parratt, Phys. Rev. 95, 359 (1954).

11 Nevot and P. Croce, Rev. Phys. Appl. 15, 761 (1980).

${ }^{12}$ V. Holy and T. Baumbach, Phys. Rev. B 49, 10668 (1994).

${ }^{13}$ D. E. Savage, J. Kleiner, N. Schimke, Y. H. Phang, T. Jankowski, J. Jacobs, R. Kariotis, and M. G. Lagalley, J. Appl. Phys. 69, 1411 (1991).

${ }^{14}$ Z. H. Ming, A. Krol, Y. L. Soo, Y. H. Kao, J. S. Park, and K. L. Wang, Phys. Rev. B 47, 16373 (1993).
${ }^{15}$ E. Spiller, D. Stearns, and M. Krumrey, J. Appl. Phys. 74, 107 (1993).

${ }^{16}$ Y. H. Phang, D. E. Savage, R. Kariotis, and M. G. Lagally, J. Appl. Phys. 74, 3181 (1993).

${ }^{17}$ E. E. Fullerton, Phys. Rev. B 45, 9292 (1992).

${ }^{18}$ T. W. Barbee Jr., SPIE 563, 2 (1985).

${ }^{19}$ F. Family, J. Phys. A: Math. Gen. 19, L441 (1986).

${ }^{20}$ D. E. Savage and N. Shimke, J. Appl. Phys. 71, 3283 (1992).

${ }^{21}$ D. E. Savage, Y. H. Phang, J. J. Rownd, J. F. Mackay, and M. G. Lagalley, J. Appl. Phys. 74, 6158 (1993).

${ }^{22}$ J. B. Kortright, J. Appl. Phys. 70, 3620 (1991).

${ }^{23}$ X. Jiang, T. H. Metzger, and J. Peisl, Appl. Phys. Lett. 61, 904 (1992).

${ }^{24}$ M. Jergel, V. Holy, E. Majaova, S. Luby, and R. Senderak, J. Phys. D 28, A241 (1995).

${ }^{25}$ X. Jiang, T. H. Metzger, and J. Peisl, Phys. Status Solidi B 179, 299 (1993). 